\title{
Reference Values for Nocturnal Home Polysomnography in Primary Schoolchildren
}

\author{
DOROTHEE MOSS, MICHAEL S. URSCHITZ, ANETTE VON BODMAN, STEFFEN EITNER, \\ ANKE NOEHREN, PILAR M. URSCHITZ-DUPRAT, MARTIN SCHLAUD, AND CHRISTIAN F. POETS \\ Department of Neonatology [D.M., M.S.U., A.V., S.E., C.F.P.], University Children's Hospital Tuebingen, \\ 72076 Tuebingen, Germany, Department of Pediatric Pulmonology and Neonatology [A.N., P.M.U.], \\ Department of Epidemiology [M.S.], Hannover Medical School, 30625 Hannover, Germany
}

\begin{abstract}
ABST
Abbreviated home polysomnography may be an alternative to
laboratory polysomnography in children but is not yet generally
accepted, partly due to a lack of reference values. Also, there are
no normative data on respiratory events obtained using nasal
prongs. We determined the prevalence and frequency of central,
obstructive, and mixed apneas and hypopneas in a population-
based sample of 50 children (mean age 10.1 years) using abbre-
viated home polysomnography and nasal prongs. We also deter-
mined the frequency of movements/arousals and body position
changes. All children had central apneas. Obstructive apneas,
mixed apneas, and hypopneas were found in 36\%, 6\%, and 14\%
of children, respectively. Average number of central, obstructive,
and mixed apneas; hypopneas; movement/arousals; and body
position changes per hour of sleep was $1.5,0.1,0.01,0.02,8.2$,
and 3.7, respectively. The corresponding cutoff values (mean
\end{abstract}
The gold standard for diagnosing sleep-disordered breathing (SDB) is full polysomnography in a sleep laboratory (1). Unattended home sleep studies using portable systems, however, are increasingly recognized as an alternative. Advantages include convenience, improved sleep quality, and costeffectiveness $(2,3)$. Nonetheless, such studies are yet rarely used in the evaluation of pediatric SDB. One reason for this may be the lack of reference values.

There are also no reference data for respiratory events in children measured by nasal prongs/pressure transducers, although these are more sensitive in detecting hypopnea and FL than thermal sensors (e.g. thermistor or thermocouples) $(4,5)$. This is important because children are more likely than adults

Received November 17, 2004; accepted March 2, 2005

Correspondence: Michael S. Urschitz, M.D., Department of Neonatology, University Children's Hospital Tuebingen, Calwerstr. 7, 72076 Tuebingen, Germany: e-mail: michael.urschitz@med.uni-tuebingen.de.

Pilar M. Urschitz-Duprat was supported by a research grant from the Hans Meineke Foundation, Hannover, Germany.

Polygraphic devices and sensors were provided by ResMed Germany (Moenchengladbach, Germany).

DOI: 10.1203/01.PDR.0000181372.34213.13 plus 2 standard deviations or 95th centile) were 3.7, 0.7, 0.1, 0.2, 13.4, and 9.1, respectively. We did not find significant gender differences regarding any sleep variable under study. The presented reference values may help clinicians and researchers to improve the interpretation of abbreviated home polysomnography in school-age children. (Pediatr Res 58: 958-965, 2005)
AHI, apnea-hypopnea index
Abbreviations
CEST, corrected estimated sleep time
D4, desaturation by $\geq 4 \% \mathrm{SpO}_{2}$
D90, desaturation to $\leq 90 \% \mathrm{SpO}_{2}$
FL, flow limitation
RDI, respiratory disturbance index
SDB, sleep-disordered breathing

to have partial rather than complete upper airway obstruction (6).

We, thus, aimed to establish reference values for respiratory and other sleep study variables obtained at home using portable devices and nasal prongs/pressure transducers. The current study was conducted as a part of a population-based crosssectional study on prevalence, risk factors, and consequences of various expressions of SDB in children (7-10). Primary school children were screened for signs and symptoms of SDB using parental questionnaires (8) and nocturnal home pulse oximetry (11). Children with and without signs and symptoms of SDB subsequently underwent nocturnal home polysomnography (8). In this report, we focus on the feasibility of performing unattended home sleep studies in children, the data quality achieved, and the presentation of reference values obtained from healthy school-age children.

\section{METHODS}

Subjects. The source population for the current study were subjects who had participated in the main study (8) and had (1) no history of habitual snoring (8), (2) an obstructive sleep apnea risk score $<0(8,12)$, and (3) an SDB risk score $<24(8,13)$. Children with abnormal oximetry results were excluded $(8)$. Of 1144 children originally participating (8), 983 children met all inclusion 
criteria. Eligible children were listed by date of enrollment to the main study, and an investigator called parents of every 20th child on the list. The course of the sleep study was explained and a ticket for the Hannover Zoo offered to the children as an incentive for participation.

Polysomnographic device. Recordings were performed overnight in the children's homes using a newly developed ambulatory polygraphic device (Embletta PDS, MedCare Flaga, Iceland). The device could be easily attached using a soft elastic belt. The montage comprised the following channels and sensors: chest and abdominal wall movements (piezo effort sensor, Pro-Tech), nasal pressure and linearized nasal airflow estimation (nasal prongs and built-in pressure transducer, MedCare Flaga), oral airflow (thermocouple, Pro-Tech), snoring (vibration sensor, New Life Technologies), arterial oxygen saturation, pulse rate, pulse wave form (pulse oximeter Xpod, Nonin Medical), actigraphy, body position and user events (all MedCare Flaga;). The nasa pressure signal was linearized by computing its square root $(14,15)$. Both the nasal pressure raw signal and the linearized airflow estimation were available The latter was used for event identification. The pulse oximeter sampled arterial oxygen saturation data on a beat-to-beat basis and delivered values in a beat-to-beat mode as well as in a four-beat exponential averaging mode; only beat-to-beat values were considered. The device included the option to record body position, provided it was calibrated in supine position at the onset of a recording. This was not appropriately achieved by all children. Thus, only changes in body position are presented.

Sleep study protocol. With institutional review board (ethics committee of the Hannover Medical School) and informed written parental consent, an investigator visited the children at home approximately 1 hour before their usual bedtime. Children were carefully examined for any acute respiratory disease or other acute health problem potentially affecting study results. If present, recordings were postponed. Sensors were carefully attached and fixed with medical tape (Fig. 1). Usually, the device was positioned in front of the thorax. If a child preferred the prone position for sleep, he or she was allowed to shift the device at the thorax's side. Handling of the device was explained to the parents and their child. A telephone hotline was set up to respond to any question or problem occurring during the night. The device was set up to start the recording automatically just before usual bedtime and to stop it at $0600 \mathrm{~h}$

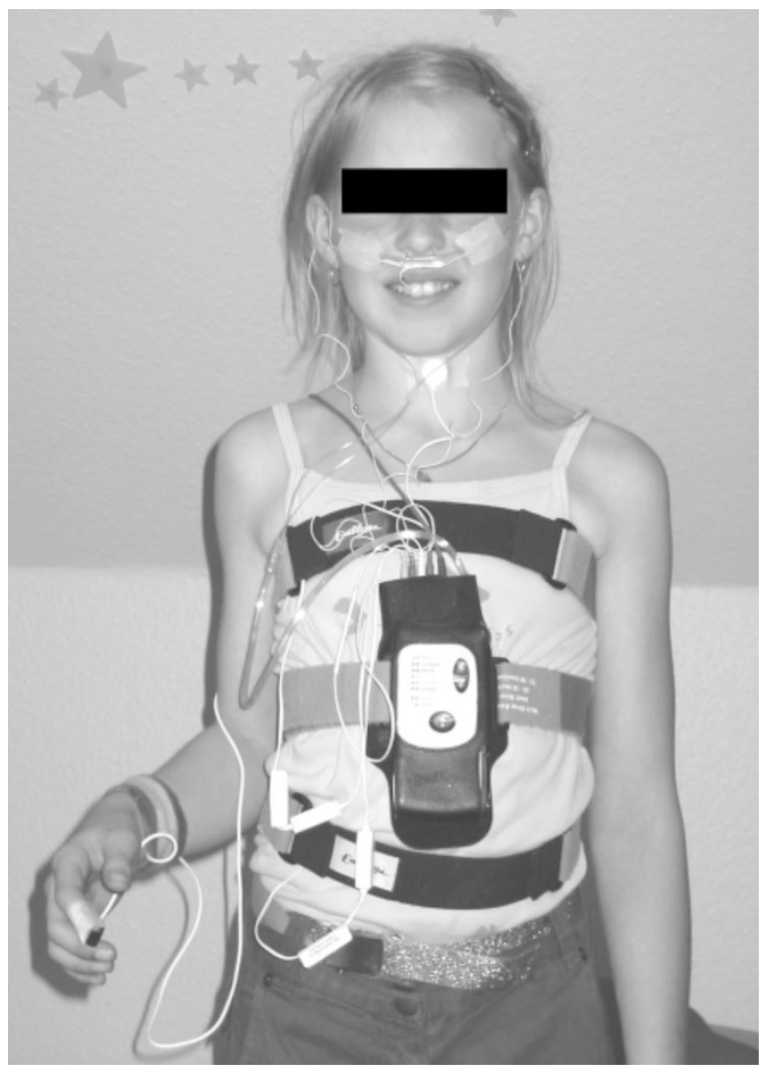

Figure 1. Polygraphic device, sensors, and fixation. The following sensors are shown (top to bottom): nasal cannula, oral flow sensor, snoring sensor (usually attached with a soft band), chest effort sensor, polygraphic device (actimeter/body position sensor/event button/pressure transducer), abdominal effort sensor, and pulse oximeter sensor (right index finger).
Children were instructed to press the event button when (i) going to bed and lying supine and (ii) lights were switched off. One of the investigators picked up the device at the child's home on the following day, downloaded the data to a PC and reviewed the recording using device-specific software (Somnologica for Embletta, version 3.1.2, MedCare Flaga). Body position and button events were then analyzed automatically. Although snoring was measured, we do not report results on this channel because widely accepted and validated scales for assessing the quality and severity of snoring in children are lacking.

Basic sleep study and signal quality variables. Recording time was determined and time in bed defined based on body position and button events. Although it is not possible to determine sleep onset exactly without EEG, a typical pattern of "calming down" can be seen in the signals. If this pattern was not interrupted by body movements for at least 10 minutes, sleep onset was assumed (Fig. 2); morning awakening was estimated as the end of the last such period. This conservative definition of sleep onset was used to avoid overestimation of sleep time and, therefore, underestimation of event indices. The estimated sleep time was calculated as the period between sleep onset and morning awakening.

Within estimated sleep time, recordings were analyzed for (i) movement periods using actigraphy, body position, and movement artifacts on other channels and (ii) artifactual or uninterpretable periods other than movement periods on either the nasal flow, thoracic effort, abdominal effort, or oximetry channel. Movement periods and artifactual/uninterpretable periods were excluded from estimated sleep time if they lasted for more than 5 minutes and the corrected estimated sleep time (i.e. estimated sleep time without movement or artifactual/uninterpretable periods, CEST) was calculated. A minimum of $4 \mathrm{~h}$ of CEST was required $(16,17)$. For infants, it has been shown that reliability of apnea estimation is adequate when recording duration exceeds 3 hours (1618). If a recording comprised less than 4 hours of CEST, the family was contacted again and asked for a repetition of the sleep study.

Recordings were then manually analyzed for central, mixed, and obstructive apneas; hypopneas; FL; arterial oxygen desaturation; and movements/arousal events based on standard guidelines or published criteria $(14,19,20)$. Typical examples are presented in Figure 3.

Respiratory events. An apnea was scored if the amplitude of the nasal airflow fell to $\leq 20 \%$ of the average amplitude of the two preceding breaths, no airflow was detected at the mouth, and the event comprised at least two breath cycles (i.e. approximately 6 seconds for the age group under study). Central apneas were scored if criteria for apnea were fulfilled and no chest and abdominal wall movements were present. Obstructive apneas were scored if criteria for apnea were fulfilled and out-of-phase movements of the chest and abdomen were present. Mixed apneas were defined as apneas with central and obstructive components, each of them lasting at least two (not necessarily consecutive) breath cycles. Hypopneas were scored if the amplitude of the nasal airflow fell to $\leq 50 \%$ of the average amplitude of the two preceding breaths, arterial oxygen desaturation of at least $4 \%$ occurred within 30 seconds of the onset of the event, and the event comprised at least two breath cycles. As no generally accepted definition for FL is available for children, an adapted definition for adults was used (14). Thus, a FL was scored if (i) the amplitude of the nasal airflow fell to $<70 \%$ of the average amplitude of the two preceding

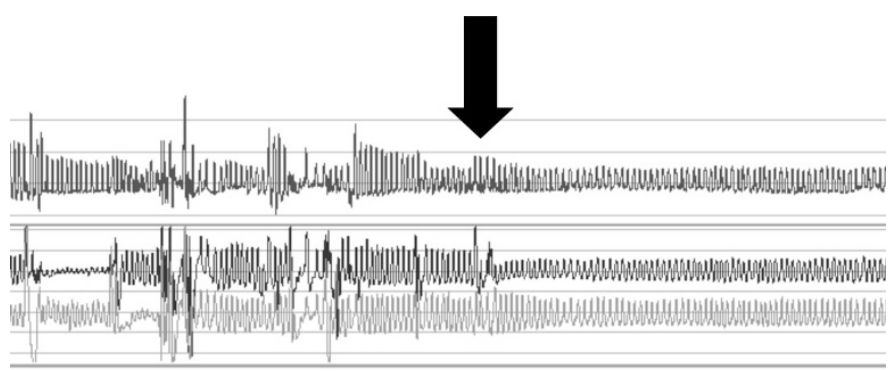

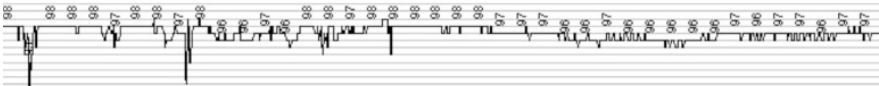

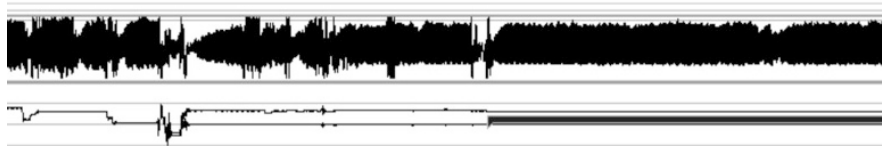

Figure 2. Sleep onset. Example for sleep onset as defined in this study (see arrow); for definition, see "Methods." Channels shown (from top to bottom): nasal airflow, chest and abdominal wall movements, arterial oxygen saturation, pulse wave form, actigraphy, and body position. 
a

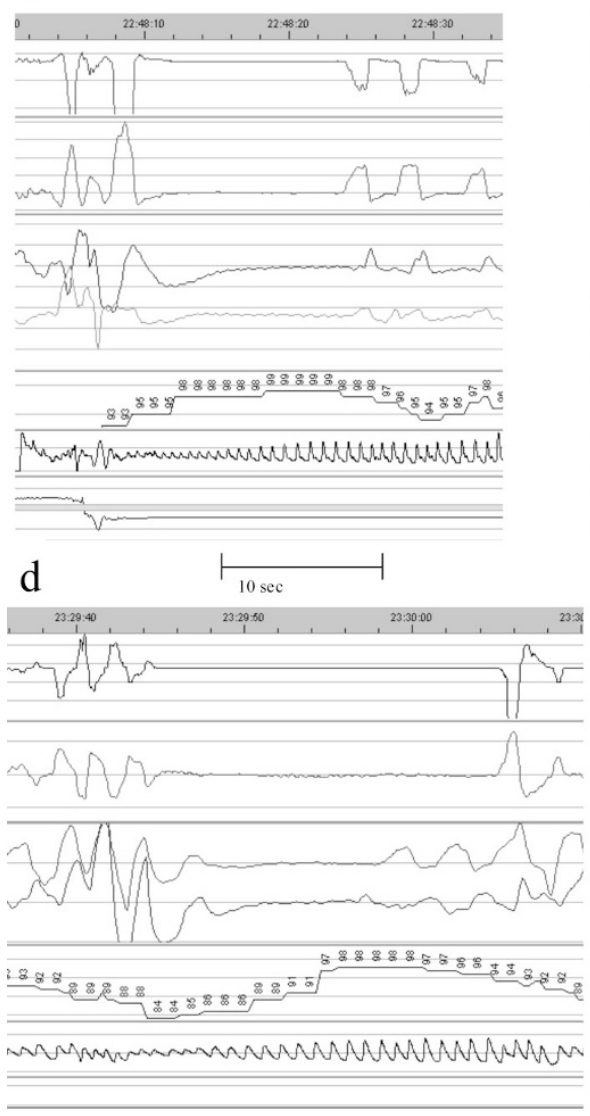

b

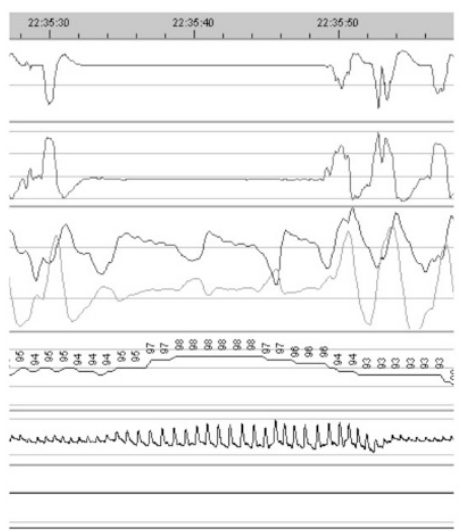

e

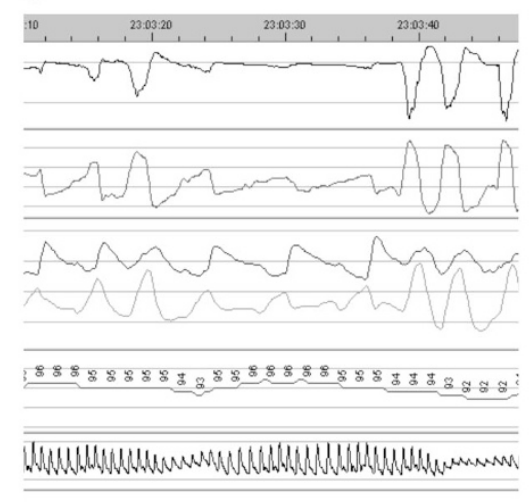

$\mathrm{C}$

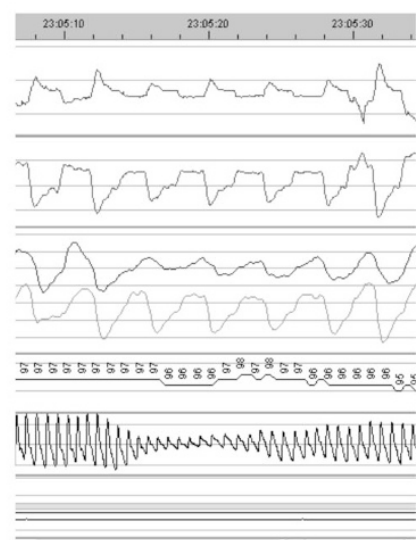

Figure 3. ( $a-e)$ Respiratory events. Typical examples for sleep study events (for definition, see "Methods"). Channels shown (from top to bottom): nasal pressure, nasal airflow, chest and abdominal wall movements, arterial oxygen saturation, pulse wave form, actigraphy, and body position. ( $a$ ) Central apnea; $(b)$ obstructive apnea; $(c)$ flow limitation; $(d)$ mixed apnea; $(e)$ hypopnea.

breaths, (ii) a flattened inspiratory nasal airflow signal appeared, (iii) the event ended abruptly with a return to breaths with higher amplitude and a more sinusoidal shape, and (iv) the event comprised at least two breath cycles (14). FL were reviewed by three of the authors and scored if all agreed on an event.

A hierarchy for scoring respiratory events was defined as follows: apnea $>$ hypopnea $>$ FL. Among apneas the hierarchy was defined as follows: mixed apnea $>$ obstructive apnea $>$ central apnea. Thus, it was not allowed to score an event as central apnea if criteria for mixed apnea were fulfilled or to score an event as FL if criteria for hypopnea were fulfilled. Peak inspiration was used to identify onset and termination of respiratory events ("peak to peak"). Two "normal" breaths terminated respiratory events. If two respiratory events were separated by only one breath, only one event was scored. The frequency and duration of all respiratory events and their association with an oxygen desaturation were determined. The longest respiratory event for each type and recording was noted. Indices, as number of events per hour of CEST, were calculated for each event type separately and for (i) central, obstructive, and mixed apneas (AI); (ii) mixed and obstructive apneas and hypopneas (MOAHI); (iii) central, obstructive, and mixed apneas and hypopneas (AHI); and (iv) central, obstructive, and mixed apneas; hypopneas; and FL (RDI).

Desaturation events. Desaturation events were visually confirmed to exclude spuriously low values. Events with a distorted pulse wave form signal before their onset were considered artifactual and excluded. The lowest oxygen saturation value and the number of desaturation events by $\geq 4 \%$ (D4) and to $\leq 90 \%$ (D90) were counted. Desaturation event indices, defined as events per hour of CEST, were calculated for D4 and D90 events.

Movement/arousal events. Movement-related changes or artifacts in two out of the following three signals were scored as movement/arousal if they lasted for 1 second or longer: (i) chest or abdominal wall movements, (ii) pulse wave form, (iii) actigraphy/body position (20). The minimal interval required between two movements/arousals was 10 seconds. A movement/arousal index, defined as number of these events per hour of CEST, was calculated. A body position change index, defined as body position changes per hour of CEST, was calculated from the results of an automated analysis (MedCare Flaga).
Inter- and intraobserver variability. To determine the applicability of the above respiratory event definitions, inter- and intraobserver agreement was assessed. Interobserver agreement was studied in four investigators and intraobserver agreement in one investigator. Years of experience in scoring polysomnographic recordings ranged from 2 to 18 among investigators. One hundred screenshots of respiratory events fulfilling or not fulfilling event definitions were selected; 20 for each of the five respiratory events (central apnea, obstructive apnea, mixed apnea, hypopnea, flow limitation event). Investigators reviewed these screenshots independently of each other and rated each event as true if criteria were fulfilled or false if these were not fulfilled. For the determination of intraobserver agreement, one investigator (D.M.) also re-reviewed the screen shots after an interval of 12 months.

Statistics. All analyses were done with statistical software (Statistical Package for the Social Science, release 11.0 for Windows; SPSS; Chicago, IL). To allow comparisons with published data, sleep study indices were expressed both as mean and standard deviation (SD) and as median, range, and interquartile range (IQR). Cutoff values for the normal range were calculated using the mean $\pm 2 \mathrm{SD}$ for normally distributed variables and the 5 th or 95 th centile for all other variables. The $\chi^{2}$ test, $t$ test, or the Mann-Whitney $U$ test were used to compare data between subgroups where appropriate. Cohen's $\kappa$ analysis was performed to assess inter- and intraobserver agreement.

\section{RESULTS}

Subjects. Of 65 children approached, 11 children or their parents refused participation. Demographic variables (gender, age, body mass index, parental education) did not differ between participants and nonparticipants. Of 54 recordings performed, 48 (89\%) comprised at least 4 hours of CEST and were thus considered sufficient for the study purpose. Two of the 
remaining recordings could be successfully repeated (one had to be repeated twice), while four children refused further participation, leading to a final sample of 50 children. Of these, three children had a history of mild respiratory allergies, one child had asthma, and one had both. None of these children were symptomatic or required treatment at the time of study. The prevalence of asthma in the study sample (4\%) was similar to the prevalence found in the underlying study population of 1144 children (5\%), while the prevalence of respiratory allergies was slightly lower in this group (6\%) than in the underlying population (11\%). Demographic data of the study sample $(n=50)$ and the underlying study population $(n=1144)$ are given in Table 1 . There was good to excellent representativeness regarding gender distribution, age, body mass index, and low socioeconomic status.

Basic sleep study and signal quality results. Only four children (8\%) reported impaired sleep quality during the night of recording. Mean (SD; range) duration of recording and estimated sleep time was $619(45 ; 474-729)$ and $542 \mathrm{~min}(50$; 397-619), respectively. Mean (SD; range) duration of movement periods and artifactual/uninterpretable periods within estimated sleep time was $8(42 ; 0-289)$ and $61 \mathrm{~min}(80$; 0-292), respectively. Thirty-nine recordings (78\%) showed artifactual/uninterpretable periods at least once in at least one channel for $\geq 5$ minutes. The nasal airflow, thoracic effort, abdominal effort, and oximetry channel was affected in $40 \%$, $14 \%, 24 \%$, and $38 \%$ of recordings, respectively. Mean proportion (SD; range) of estimated sleep time with artifactual/ uninterpretable periods in the nasal airflow, thoracic effort, abdominal effort, and the oximetry channel was 9.5 (15.2; $0-55.8), 1.7$ (6.2; 0-40.4), 9.0 (24.9; 0-100), and 3.8\% (9.1; $0-53.1$ ), respectively. Mean proportion (SD; range) of estimated sleep time with artifactual/uninterpretable periods simultaneously in the thoracic and abdominal effort channel was $0.6 \%$ (3.3; 0-22.6). Mean (SD; range) proportion of estimated sleep time with acceptable signal quality was $89 \%$ (14.2; 45.4-100). Finally, mean (SD; range) duration of CEST was 483 min $(89 ; 243-628)$. There were no statistically significant differences between boys and girls regarding basic sleep study and signal quality results.

Respiratory events. Descriptive statistics for respiratory events are presented in Table 2. In total, there were 599 central apneas, 316 in boys and 283 in girls. Two hundred twenty-two central apneas $(36.9 \%)$ were followed by a D4, but only 20 central apneas $(3.3 \%)$ were associated with a D90. Forty children (80.0\%) had central apneas associated with a D4, but only nine $(18 \%)$ had central apneas followed by a D90. Thirteen children (26\%) had central apneas of more than 20-second duration, but only one child presented a single central apnea lasting more than 25 seconds ( 30.5 seconds). There was a total of 42 obstructive apneas (21 in boys), with a maximum of nine in the recording of the child with asthma and respiratory allergy (house dust mite). Twelve obstructive apneas (28.6\%) were associated with a D4, but none was followed by a D90. Four mixed apneas occurred in three recordings; three were associated with a D4, but none was followed by a D90. The number of hypopneas totaled 10; corresponding to our definitions, all were followed by a D4.

FL events were more frequent than hypopneas (a total of 48 events) and were found more often in boys (30.8\% versus $4.2 \%$ of girls; $p<0.05$ ); none was followed by a $\mathrm{D} 4$ or $\mathrm{D} 90$. Average duration (SD) of FL was 48.9 (25.9) seconds. Although we had no maximum duration criterion for FL, no event lasted $>3$ minutes. Average duration (SD) of longest FL was 67.3 (40.3) seconds. A respiratory disturbance index (RDI) was calculated as central, obstructive, and mixed apnea plus hypopnea and FL events per hour of CEST. Mean (SD; range) RDI was 1.8 (1,4; 0.1-7.5). Recommended reference value for RDI including FL events is 4.6 per hour CEST. Apart from clear FL events, we also found periods with continuously flattened airflow signal lasting $>3$ minutes, suggesting increased upper airway resistance. These periods, however, did not start with a reduction in flow amplitude and were thus not scored as FL events.

Descriptive statistics for respiratory event indices are given in Table 3. Except for FL, there was no statistically significant gender difference regarding any event index under study. Therefore, results were not stratified by gender. As the clinical significance of such events remains unknown, no reference values are presented for FLs.

Desaturation events. A total of 308 D4 events occurred in 43 recordings (86\%). Most D4 events $(n=239 ; 78 \%$ of all D4) were associated with respiratory events. There were 69 isolated D4 events, i.e. not accompanied by a respiratory event. Only 15 D90 events were present in nine subjects (18\%), all associated with central apnea. The lowest oxygen saturation value found was $83 \%$ and associated with a central apnea. One child, suffering from asthma, presented with $35 \mathrm{D} 4$. This child also had the highest number of central apnea $(n=49)$. Another child presented with 38 D4 and four D90 events. This child had

Table 1. Comparisons between study sample and study population

\begin{tabular}{|c|c|c|c|c|c|c|c|}
\hline \multirow[b]{2}{*}{ Characteristic } & \multirow[b]{2}{*}{ Definition } & \multicolumn{3}{|c|}{ Study population } & \multicolumn{3}{|c|}{ Study sample } \\
\hline & & $\begin{array}{c}\text { Total } \\
(n=1144)\end{array}$ & $\begin{array}{c}\text { Boys } \\
(n=585)\end{array}$ & $\begin{array}{c}\text { Girls } \\
(n=559)\end{array}$ & $\begin{array}{c}\text { Total } \\
(n=50)\end{array}$ & $\begin{array}{c}\text { Boys } \\
(n=26)\end{array}$ & $\begin{array}{c}\text { Girls } \\
(n=24)\end{array}$ \\
\hline Boys & $\mathrm{N}(\%)$ & $585 \quad(51.1)$ & & & $26 \quad(52.0)$ & & \\
\hline Age at study enrollment, y & Mean (SD) & $9.6(0.7)$ & $9.7(0.7)$ & $9.5(0.6)$ & $9.3(0.7)$ & $9.3(0.7)$ & $9.3(0.6)$ \\
\hline Age at sleep study, y & Mean (SD) & & & & $10.1(0.7)$ & $10.2(0.7)$ & $9.9(0.7)$ \\
\hline Body mass index at study enrollment $\left(\mathrm{kg} / \mathrm{m}^{2}\right)$ & Mean (SD) & $17.5(2.9)$ & $17.8(3.1)$ & $17.2(2.8)$ & $16.9(2.1)$ & $16.9(2.2)$ & $16.9(2.2)$ \\
\hline Body mass index at sleep study $\left(\mathrm{kg} / \mathrm{m}^{2}\right)$ & Mean (SD) & & & & $16.8(2.1)$ & $16.8(2.1)$ & $16.9(2.2)$ \\
\hline Low socioeconomic status* & No. $(\%)$ & $285 \quad(24.9)$ & 139 (23.8) & $146 \quad(26.1)$ & $9 \quad(20.0)$ & $5 \quad(19.2)$ & $4 \quad(16.7)$ \\
\hline
\end{tabular}

* Defined as highest graduation from school on a 4-point scale, with the lowest level present in the mother.

SD, standard deviation. 
Table 2. Descriptive statistics for respiratory sleep study variables in the total study sample

\begin{tabular}{|c|c|c|}
\hline Respiratory variable & Definition & $\begin{array}{l}\text { Total study sample } \\
\quad(n=50)\end{array}$ \\
\hline No. of children with central apnea & No. $(\%)$ & $50 \quad(100)$ \\
\hline Average duration of central apnea, $s$ & Mean (SD) & $12.7(1.9)$ \\
\hline No. of children with obstructive apnea & No. $(\%)$ & $18 \quad(36)$ \\
\hline Average duration of obstructive apnea, $\mathrm{s}$ & Mean (SD) & $14.1(3.1)$ \\
\hline Duration of longest obstructive apnea per recording, $\mathrm{s}$ & Mean (SD) & $16.3(6.9)$ \\
\hline Duration of longest mixed apnea per recording, s & Mean (SD) & $13.6(4.5)$ \\
\hline No. of children with hypopnea & No. $(\%)$ & 7 (14) \\
\hline Average duration of hypopnea, s & Mean (SD) & $12.7(4.0)$ \\
\hline Duration of longest hypopnea per recording, s & Mean (SD) & $13.6(4.5)$ \\
\hline
\end{tabular}

SD, standard deviation.

Table 3. Descriptive statistics and reference values for respiratory, desaturation, and movement/arousal variables

\begin{tabular}{|c|c|c|c|c|c|c|}
\hline Sleep study variable & \multicolumn{6}{|c|}{ Total study sample $(n=50)$} \\
\hline No. of CA/h of CEST & 1.5 & $(1.1)$ & $\frac{\text { Median }}{1.3}$ & $\frac{\mathrm{IQR}}{0.7-2.0}$ & $\begin{array}{c}\text { Range } \\
0.1-5.1\end{array}$ & $\frac{\text { Reference value }}{3.7^{*}}$ \\
\hline No. of CA associated with D90/h of CEST & 0.05 & $(0.1)$ & 0.0 & $0.0-0.0$ & $0.0-0.6$ & $0.5^{\dagger}$ \\
\hline $\mathrm{SpO}_{2}$ nadir following $\mathrm{CA}$ & 92.6 & $(3.0)$ & 93.0 & $91.0-95.0$ & $83.0-97.0$ & $86.6^{*}$ \\
\hline No. of OA/h of CEST & 0.1 & $(0.2)$ & 0.0 & $0.0-0.1$ & $0.0-1.2$ & $0.7^{\dagger}$ \\
\hline No. of MA/h of CEST & 0.01 & $(0.04)$ & 0.0 & $0.0-0.0$ & $0.0-0.2$ & $0.14^{\dagger}$ \\
\hline No. of MA associated with D4/h of CEST & 0.007 & $(0.04)$ & 0.0 & $0.0-0.0$ & $0.0-0.2$ & $0.06^{\dagger}$ \\
\hline $\mathrm{SpO}_{2}$ nadir following MA & 94.7 & $(2.1)$ & 94.0 & $93.0-97.0$ & $93.0-97.0$ & $90.5^{*}$ \\
\hline No. of $\mathrm{H} / \mathrm{h}$ of CEST & 0.02 & $(0.06)$ & 0.0 & $0.0-0.0$ & $0.0-0.2$ & $0.2^{\dagger}$ \\
\hline $\mathrm{SpO}_{2}$ nadir following $\mathrm{H}$ & 93.4 & $(1.0)$ & 93.0 & $93.0-94.0$ & $92.0-95.0$ & $91.4^{*}$ \\
\hline No. of D4/h of CEST & 0.7 & $(0.9)$ & 0.5 & $0.2-0.9$ & $0.0-4.6$ & $2.5^{*}$ \\
\hline No. of CA plus OA plus MA/h of CEST (AI) & 1.6 & $(1.1)$ & 1.4 & $0.7-2.0$ & $0.1-5.5$ & $3.8^{*}$ \\
\hline No. of OA plus MA plus H/h of CEST (MOAHI) & 0.1 & $(0.3)$ & 0.0 & $0.0-0.2$ & $0.0-1.2$ & $0.8^{\dagger}$ \\
\hline No. of CA plus OA plus MA plus H/h of CEST (AHI) & 1.6 & $(1.2)$ & 1.4 & $0.7-2.0$ & $0.1-5.7$ & $4.0^{*}$ \\
\hline
\end{tabular}

* Variable is normally distributed; recommended value is calculated as mean \pm double standard deviation.

$\dagger$ Variable is not normally distributed; recommended value is calculated as the 5th or 95th centile.

$\mathrm{SD}$, standard deviation; IQR, interquartile range; CEST, corrected estimated sleep time; CA, central apnea; OA, obstructive apnea; MA, mixed apnea; H, hypopnea; $\mathrm{SpO}_{2}$, pulse oximeter oxygen saturation; D4, desaturation by $\geq 4 \% \mathrm{SpO}_{2}$; D90, desaturation to $\leq 90 \% \mathrm{SpO}_{2}$; $\mathrm{AI}$, apnea index, $\mathrm{MOAHI}$, mixed obstructive apnea-hypopnea index; AHI, apnea-hypopnea index.

parent-reported "chronic bronchitis." Descriptive statistics for desaturation event indices are presented in Table 3.

Movement/arousal events. At least one change in body position was present in 49 recordings $(98 \%)$. The mean (SD; range) number of body position changes per recording was 27.5 (16.2; $0-86)$. One child remained in supine position for the entire night, whereas another had 86 changes in body position. Both children had no abnormal values in any other variable. Movements/arousals were present in all recordings, with a mean (SD; range) number of $65.7(24.5 ; 24-134)$ per recording. Descriptive statistics for changes in body position and movement/arousal indices are given in Table 3.

Inter- and intraobserver variability. Assessment of interobserver variability revealed an overall good agreement (Cohen's $\kappa$; range: $0.68-0.82$; all $p<0.001$ ). Subgroup analyses showed poorest agreement for hypopnea (Cohen's $\kappa$; mean/range: $0.5 /$ 0.29-0.83), moderate agreement for mixed apnea (Cohen's $\kappa$; mean/range: 0.64/0.48-1.0), FL events (Cohen's $\kappa$; mean/range: 0.65/0.51-0.8) and central apnea (Cohen's $\kappa$; mean/range: 0.69/ 0.5-0.89), and excellent agreement for obstructive apnea (Cohen's $\kappa$; mean/range: 0.82/0.73-0.86). Assessment of intraobserver variability showed good agreement (Cohen's $\kappa$ : $0.8 ; p<$ 0.001 ). Agreement was best for mixed apnea (Cohen's $\kappa: 0.83 ; p$ $<0.001$ ) and again poorest for hypopnea (Cohen's $\kappa: 0.57 ; p=$ $0.01)$. There was no difference regarding Cohen's $\kappa$ between less and more experienced observers (data not shown).

\section{DISCUSSION}

Using a new ambulatory recording device, we found nocturnal home sleep studies, performed in a research setting, sufficiently acceptable and feasible to obtain interpretable data on respiration, oxygen saturation, and sleep disruption in 50 out of 53 primary schoolchildren. Our study sample (popula- 
tion-based cohort), setting (the children's homes), and methods (nasal pressure transducer and beat-to-beat pulse oximetry) enabled us to obtain valid and comparatively representative reference values for the variables under study. These reference data may help to improve the interpretation of sleep studies in school-age children.

Several authors have reported pediatric reference values for sleep variables (21-28). These data clearly demonstrated that adult criteria and reference values for sleep and respiratory variables cannot be transferred to children. During the past decade, several studies determined the frequency of obstructive and mixed apneas, hypoventilation, and hypopnea in children $(23,27,28)$, but all had similar limitations. They were not population based (and thus subject to selection bias), were performed in sleep labs, used inaccurate sensor techniques for the detection of hypopnea, and did not report on FL events. Thus, further studies on reference values were required.

In contrast to adults, in whom an $\mathrm{AHI} \geq 5$ is often used to diagnose SDB (29), the AHI is not generally accepted to diagnose SDB in children. The obstructive apnea index or reference values for hypoventilation are often used instead (23). This is partly due to a lack of generally accepted event definitions, measurement techniques, and reference data for hypopnea in children but is nevertheless surprising given that partial upper airway obstruction is more common in children than in adults (6). Relying solely on gas exchange abnormalities to detect these events may lead to an underestimation of their frequency, particularly with short events (30). Only two studies reported reference values for hypopnea in children $(24,27)$. These data, however, were obtained using end-tidal carbon dioxide monitors or thermal sensors, which are not generally recommended for hypopnea detection (29). Recent studies in adults $(31)$ and children $(4,5)$ revealed an inferior performance of thermal sensors compared with nasal pressure measurements in detecting hypopnea. Reference values for hypopnea using this new technique are now available and support other reports that hypopneas are rare in children $(24,27)$.

Since their first description in 1994 (32), FL events based on the contour of the inspiratory flow tracing are increasingly recognized in adults. Their presence indicates residual upper airway resistance both during mechanical ventilation (32) and regular breathing (33). Respiratory disturbance indices including FL events obtained from nasal prongs and pressure transducers were found to discriminate better between symptomatic and asymptomatic SDB than classic apnea-hypopnea indices (14). Also in children, FL events may be associated with increased esophageal pressure swings, indicating increased upper airway resistance $(4,5)$. Thus, FL events may play similar roles in children and adults. We found FL events in $18 \%$ of subjects, predominantly boys. This male preponderance remains unexplained but may indicate early gender differences in mild obstructive events. The clinical significance of these events, however, has yet to be shown. Despite this, the presented reference values for FL events may supplement prior reference data for other measures of partial upper airway obstruction, which are more difficult to use in the home setting (e.g. end-tidal carbon dioxide measurements).
Several researchers used commercially available polygraphic devices for sleep studies at home, all in research settings (16,34-37). Acceptance was good to excellent in most studies. In the TuCASA study, only $6 \%$ of children stated that they had slept worse during the study night (16). Feasibility data demonstrated an initial pass rate of $91 \%$ and a final pass rate of $94-97 \%$ for home sleep recordings $(16,37)$. In our study, $8 \%$ of children reported impairments in sleep quality. Although we found periods with impaired signal quality in about $70 \%$ of recordings, we obtained $>4$ hours of interpretable signals in $89 \%$ at first pass, improving to $93 \%$ after repetition. These results confirm those of others, who performed unattended polysomnography in adults (38) and children between 5 and 8 years of age (16) and demonstrated that unattended sleep studies can be obtained with sufficient quality at home in school-age children. Further studies are needed to reveal whether unattended studies could also be an option for younger children.

Nasal pressure measurements, however, have not yet been used routinely in children and in particular not at home. Disadvantages include an increased amount of uninterpretable signal $(4,16)$, occurring with sensor dislocation or occlusion, or during periods of mouth breathing (39). In the present study, one third of children had at least 5 minutes of uninterpretable nasal pressure signal. Some authors suggest discarding such periods (40), and others regard recurrent mouth-breathing periods as abnormal respiration resulting in increased respiratory effort (41). Unfortunately, there is yet no standard definition for assessment of mouth breathing. As we were primarily interested in respiratory events detected by nasal prongs, we decided to discard such periods. Children with SDB, however, frequently present with mouth breathing leading to even more uninterpretable signal periods. This remains a major concern.

Arousal from sleep is usually scored based on electroencephalographic criteria. Specific reference values are available for children $(20,24,26)$. Unfortunately, only about one third of respiratory events in children are terminated by cortical arousal (42). Thus, scoring of movement/arousal (20) or subcortical arousal (43) has been suggested as an alternative. These types of arousal accompanied $71-83 \%$ of respiratory events $(20,43)$ and may thus be more sensitive markers for respiratory-related sleep disruption in children. Data on the frequency of movements/arousals in children have not been reported before. Stores and colleagues (26) reported a mean cortical arousal index of 8.5 for 8 - to 9-year-old children, which is similar to our data. Whether our definition increases the sensitivity to respiratory-related sleep disruption remains to be shown. It certainly has the advantage of not requiring electroencephalography (EEG).

We found the 95th centile for the obstructive apnea index to be 0.7 , which supports prior suggestions that obstructive apnea is rare in children (28). The cutoff value for the obstructive apnea index suggested from our data is higher than that reported by Uliel et al. (28), and slightly lower than that reported by Marcus et al. (23). In contrast to the latter, we did not use EEG to differentiate sleep from wakefulness. One concern with omitting EEG in cardiopulmonary polysomnography is loss of diagnostic accuracy. This is based on the assumption that if 
detection of rapid eye movement sleep (when SDB is usually present or most severe) is not possible, SDB cannot be reliably ruled out. However, as previously discussed by Jacob et al. (2) and Morielli et al. (44), there is invariably REM sleep present in an all-night recording, even though it may not be possible to determine which specific epochs are included.

Central apneas are common in healthy children (22). Some authors, however, consider them abnormal if associated with a desaturation to $<90 \%$ (23) or lasting for $>20$ seconds (22). We found short central apneas in all recordings, and those lasting $>20$ seconds in one third, whereas associations with desaturation $\leq 90 \%$ occurred in only two subjects $(3 \%)$. Thus, central apneas of up to 30-second duration may be common in children. This is in contrast to previous studies (22) and may be related to our apnea definition, which required at least two breaths to terminate an apnea. This underscores yet again that reference values are only valid for the event definitions used. This also applies to desaturation events in which our results are comparable to those of previous work from our group also using a pulse oximeter in beat-to-beat mode (45), but are considerably higher than those reported for other instruments using variable averaging times (11).

Limitations. Although our sample was selected from a population-based cohort of primary schoolchildren, it was formed using a series of exclusion criteria. It is thus not fully representative of the initial cohort, although the prevalence of asthma and allergies was similar. Results may therefore reflect the occurrence of respiratory events in healthy children rather than in an unselected sample of schoolchildren. It should also be stressed that the most frequent ages of children referred to pediatric sleep laboratories for evaluation of SDB are preschoolers. The current study, however, included only schoolage children. The reference values obtained should thus only be applied to this age group. Although we tried to limit our analyses to sleep periods, we were unable to fully exclude periods of wakefulness because EEG was not used. As mentioned above, this likely biased our results toward lower values (assuming that respiratory events are rarer when awake). Thus, we explicitly do not recommend using these reference values for full polysomnography.

Another point of concern is intra- and interobserver variability. Our results show good agreement between trained scorers, but there was still disagreement, particularly in scoring hypopnea and mixed apnea. Even intraobserver agreement was poor for scoring hypopnea. This may be related to the event definitions used. For example, it is not always possible to determine the degree of amplitude reduction precisely with manual analysis. Automatic analysis of respiratory events would eliminate this problem, but data on the sensitivity and specificity of such tools are lacking. Further studies on intra- and interobserver variability and, if available, improved automated analysis for respiratory events in polysomnography are needed.

The device used in this study was usually attached to the children's front. In children preferring the prone sleep position, however, it was attached to the right or left flank. Nevertheless, an uncomfortable sleep position during the recording may have modified normal sleep and breathing behavior, potentially influencing recording results.

\section{CONCLUSION}

Our data suggest that abbreviated cardiopulmonary sleep studies may be successfully performed at home in $>90 \%$ of school-age children. The method was well accepted by both parents and children and generated valid information on nocturnal breathing and respiratory events. Central apnea was common in these children, while obstructed breathing episodes were rare. In healthy children, nighttime desaturations events to $\leq 90 \%$ were consequences of central apneas but not of obstructed breathing episodes. Except for FL events, no gender differences were present in our sample. These reference data may help clinicians and researchers to improve the interpretation of sleep studies in school-age children.

Acknowledgments. The authors thank Dr. Ehrhardt (Department of Public Health, City Council, Hannover, Germany), Mrs. Martinsen (Supervisory School Authority, Hannover, Germany), Mr. Hegemann (District Government, Hannover, Germany), and the headmasters and teachers of the participating schools for their support and cooperation. Thanks also go to Mr. Sprenger from ResMed Germany (Moenchengladbach, Germany) for technical assistance and support. The authors thank the Hans Meineke Foundation (Hannover, Germany) and Mrs. Waszulewski from the Hannover Zoo for supporting this study and particularly thank all the children and their parents for their patience and cooperation; they made this study possible.

\section{REFERENCES}

1. American Academy of Pediatrics, Section on Pediatric Pulmonology, Subcommittee on Obstructive Sleep Apnea Syndrome 2002 Clinical practice guideline: diagnosis and management of childhood obstructive sleep apnea syndrome. Pediatrics 109:704712

2. Jacob SV, Morielli A, Mograss MA, Ducharme FM, Schloss MD, Brouillette RT 1995 Home testing for pediatric obstructive sleep apnea syndrome secondary to adenotonsillar hypertrophy. Pediatr Pulmonol 20:241-252

3. Parra O, Garcia-Esclasans N, Montserrat JM, Garcia Eroles L, Ruiz J, Lopez JA, Guerra JM, Sopena JJ 1997 Should patients with sleep apnoea/hypopnoea syndrome be diagnosed and managed on the basis of home sleep studies? Eur Respir J 10:1720-1724

4. Trang H, Leske V, Gaultier C 2002 Use of nasal cannula for detecting sleep apneas and hypopneas in infants and children. Am J Respir Crit Care Med 166:464-468

5. Serebrisky D, Cordero R, Mandeli J, Kattan M, Lamm C 2002 Assessment of inspiratory flow limitation in children with sleep-disordered breathing by a nasal cannula pressure transducer system. Pediatr Pulmonol 33:380-387

6. Marcus CL 2000 Obstructive sleep apnea syndrome: differences between children and adults. Sleep 23(suppl 4):S140-S141

7. Urschitz MS, Guenther A, Eggebrecht E, Wolff J, Urschitz-Duprat PM, Schlaud M, Poets CF 2003 Snoring, intermittent hypoxia and academic performance in primary school children. Am J Respir Crit Care Med 168:464-468

8. Schlaud M, Urschitz MS, Urschitz-Duprat PM, Poets CF 2004 The German study on sleep-disordered breathing in primary school children: epidemiological approach, representativeness of study sample, and preliminary screening results. Pediatr Perinat Epidemiol 18:431-440

9. Urschitz MS, Eitner S, Guenther A, Eggebrecht E, Wolff J, Urschitz-Duprat PM, Schlaud M, Poets CF 2004 Habitual snoring, intermittent hypoxia, and impaired behavior in primary school children. Pediatrics 114:1041-1048

10. Urschitz MS, Guenther A, Eitner S, Urschitz-Duprat PM, Schlaud M, Ipsiroglu OS, Poets CF 2004 Risk factors and natural history of habitual snoring. Chest 126:790800

11. Urschitz MS, Wolff J, Von Einem V, Urschitz-Duprat PM, Schlaud M, Poets CF 2003 Reference values for nocturnal home pulse oximetry during sleep in primary school children. Chest 123:96-101

12. Brouilette R, Hanson D, David R, Klemka L, Szatkowski A, Fernbach S, Hunt C 1984 A diagnostic approach to suspected obstructive sleep apnea in children. J Pediatr 105:10-14

13. Gozal D 1998 Sleep-disordered breathing and school performance in children. Pediatrics 102:616-620

14. Hosselet J, Ayappa I, Norman RG, Krieger AC, Rapoport DM 2001 Classification of sleep-disordered breathing. Am J Respir Crit Care Med 163:398-405 
15. Montserrat JM, Farre R, Ballester E, Felez MA, Pasto M, Navajas D 1997 Evaluation of nasal prongs for estimating nasal flow. Am J Respir Crit Care Med 155:211-215

16. Goodwin JL, Enright PL, Kaemingk KL, Rosen GM, Morgan WJ, Fregosi RF, Quan SF 2001 Feasibility of using unattended polysomnography in children for research-report of the Tucson Children's Assessment of Sleep Apnea study (TuCASA). Sleep 24:937-944

17. Simakajornboon N, Beckerman RC, Mack C, Sharon D, Gozal D 2002 Effect of supplemental oxygen on sleep architecture and cardiorespiratory events in preterm infants. Pediatrics 110:884-888

18. Duke JC, Stahl ML, Parrish WE, Rundell OH, Orr WC 1984 Predicting sleep apnea rates in infants. Sleep Res 13:201

19. American Thoracic Society 1996 Standards and indications for cardiopulmonary sleep studies in children. Am J Respir Crit Care Med 153:866-878

20. Mograss MA, Ducharme FM, Brouillette RT 1994 Movement/arousals. Description, classification, and relationship to sleep apnea in children. Am J Respir Crit Care Med 150:1690-1696

21. Guilhaume A, Benoit O 1976 [Respiratory pauses during sleep in the normal child. Observation of 3 pathological cases]. Rev Electroencephalogr Neurophysiol Clin 6:116-123

22. Carskadon MA, Harvey K, Dement WC, Guilleminault C, Simmons FB, Anders TF 1978 Respiration during sleep in children. West J Med 128:477-481

23. Marcus CL, Omlin KJ, Basinki DJ, Bailey SL, Rachal AB, Von Pechmann WS, Keens TG, Ward SL 1992 Normal polysomnographic values for children and adolescents. Am Rev Respir Dis 146:1235-1239

24. Acebo C, Millman RP, Rosenberg C, Cavallo A, Carskadon MA 1996 Sleep, breathing, and cephalometrics in older children and young adults. Part I-normative values. Chest 109:664-672

25. Stores G, Crawford C, Selman J, Wiggs L 1998 Home polysomnography norms fo children. Technol Health Care 6:231-236

26. Stores G, Crawford C 2000 Arousal norms for children age 5-16 years based on home polysomnography. Technol Health Care 8:285-290

27. Witmans MB, Keens TG, Davidson Ward SL, Marcus CL 2003 Obstructive hypopneas in children and adolescents: normal values. Am J Respir Crit Care Med 168:1540

28. Uliel S, Tauman R, Greenfeld M, Sivan Y 2004 Normal polysomnographic respiratory values in children and adolescents. Chest 125:872-878

29. American Academy of Sleep Medicine Task Force 1999 Sleep-related breathing disorders in adults: recommendations for syndrome definition and measurement techniques in clinical research. Sleep 22:667-689

30. Guilleminault C, Pelayo R, Leger D, Clerk A, Bocian RC 1996 Recognition of sleep-disordered breathing in children. Pediatrics 98:871-882

31. Norman RG, Ahmed MM, Walsleben JA, Rapoport DM 1997 Detection of respiratory events during NPSG: nasal cannula/pressure sensor versus thermistor. Sleep $20: 1175-1184$
32. Condos R, Norman RG, Krishnasamy I, Peduzzi N, Goldring RM, Rapoport DM 1994 Flow limitation as a noninvasive assessment of residual upper-airway resistance during continuous positive airway pressure therapy of obstructive sleep apnea. Am J Respir Crit Care Med 150:475-480

33. Clark SA, Wilson CR, Satoh M, Pegelow D, Dempsey JA 1998 Assessment of inspiratory flow limitation invasively and noninvasively during sleep. Am J Respir Crit Care Med 158:713-722

34. Brunetti L, Rana S, Lospalluti ML, Pietrafesa A, Francavilla R, Fanelli M, Armenio L 2001 Prevalence of obstructive sleep apnea syndrome in a cohort of 1,207 children of southern Italy. Chest 120:1930-1935

35. Sanchez-Armengol A, Fuentes-Pradera MA, Capote-Gil F, Garcia-Diaz E, CanoGomez S, Carmona-Bernal C, Castillo-Gomez J 2001 Sleep-related breathing disorders in adolescents aged 12 to 16 years: clinical and polygraphic findings. Chest 119:1393-1400

36. Castronovo V, Zucconi M, Nosetti L, Marazzini C, Hensley M, Veglia F, Nespoli L, Ferini-Strambi L 2003 Prevalence of habitual snoring and sleep-disordered breathing in preschool-aged children in an Italian community. J Pediatr 142:377-382

37. Rosen CL, Larkin EK, Kirchner HL, Emancipator JL, Bivins SF, Surovec SA, Martin RJ, Redline S 2003 Prevalence and risk factors for sleep-disordered breathing in 8- to 11-year-old children: association with race and prematurity. J Pediatr 142:383-389

38. Kapur VK Rapoport DM, Sanders MH, Enright P, Hill J, Iber C, Romaniuk J 2000 Rates of sensor loss in unattended home polysomnography: the influence of age, gender, obesity, and sleep-disordered breathing. Sleep 23:682-688

39. Hernandez L, Ballester E, Farre R, Badia JR, Lobelo R, Navajas D, Montserrat JM 2001 Performance of nasal prongs in sleep studies: spectrum of flow-related events. Chest 119:442-450

40. Montserrat JM, Farre R 2002 Breathing flow disturbances during sleep: can they be accurately assessed by nasal prongs? Am J Respir Crit Care Med 166:259-260

41. Series F, Marc I 1999 Nasal pressure recording in the diagnosis of sleep apnoea hypopnoea syndrome. Thorax 54:506-510

42. McNamara F, Issa FG, Sullivan CE 1996 Arousal pattern following central and obstructive breathing abnormalities in infants and children. J Appl Physiol 81:26512657

43. Katz ES, Lutz J, Black C, Marcus CL 2003 Pulse transit time as a measure of arousal and respiratory effort in children with sleep-disordered breathing. Pediatr Res 53:580-588

44. Morielli A, Desjardins D, Brouillette RT 1993 Transcutaneous and end-tidal carbon dioxide pressures should be measured during pediatric polysomnography. Am Rev Respir Dis 148:1599-1604

45. Poets CF, Stebbens VA, Samuels MP, Southall DP 1993 Oxygen saturation and breathing patterns in children. Pediatrics 92:686-690 